\title{
Abordagem ventilatória protetora no tratamento da hérnia diafragmática congênita
}

\author{
Gentle ventilatory approach for the treatment of congenital diaphragmatic hernia
}

Felipe de Souza Rossi ${ }^{1}$, Arno Norberto Warth ${ }^{2}$,Alice D'Agostini Deutsch ${ }^{3}$, Eduardo Juan Troster ${ }^{4}$, Celso Moura Rebello ${ }^{5}$

\section{RESUMO}

Objetivo: Descrever a evolução de recém-nascidos com diagnóstico de hérnia diafragmática congênita admitidos na Unidade de Terapia Intensiva Neonatal de um hospital privado de nível terciário, no qual aplicou-se uma estratégia ventilatória protetora.

Métodos: Coorte histórica com análise de prontuários de pacientes portadores de hérnia diafragmática congênita, admitidos de junho de 2001 a julho de 2006. Avaliaramse dados referentes ao recém-nascido (índices prognósticos antenatais, peso ao nascimento, idade gestacional, sexo), dados da reanimação e estabililização pré-operatória, cuidados pós-operatórios e taxa de sobrevida.

Resultados: Oito neonatos tiveram diagnóstico de hérnia diafragmática congênita. $O$ peso variou entre 2,38 e 3,45kg e a idade gestacional, entre 36 e 39 semanas; cinco deles eram do sexo masculino. Todos foram intubados em sala de parto até o final do primeiro minuto de vida. A correção cirúrgica ocorreu entre o segundo e o sexto dias de vida e, em quatro pacientes, houve necessidade do uso de patch. Uma estratégia ventilatória protetora foi utilizada em seis neonatos, com dados gasométricos visando $\mathrm{PaO}_{2}$ pré-ductal normal e tolerando-se hipercapnia $\left(\mathrm{PaCO}_{2} 50 \mathrm{a} 60 \mathrm{mmHg}\right)$. A extubação ocorreu entre o primeiro e o $12^{\circ}$ dias do pósoperatório, com exceção de um paciente. Seis recém-nascidos receberam alta, em média, com 30 dias de vida (19a 55 dias). A sobrevida foi de $75 \%$.

Conclusões: A sistematização do cuidado de pacientes com hérnia diafragmática congênita pode garantir, em nosso meio, uma sobrevida comparável aos principais centros mundiais que lidam com a doença.

Palavras-chave: hérnia diafragmática; respiração artificial; recém-nascido.

\section{ABSTRACT}

Objective: To describe the clinical evolution of newborns with congenital diaphragmatic hernia admitted to neoretal Intensive Care Unit of a tertiary private hospital and treated with a gentle ventilatory approach.

Methods: Analysis of charts of patients born between June 2001 and July 2006. The following data were collected: birth weight, gestational age, sex, delivery room procedures, pre and post-surgery parameters and survival rate.

Results: Eight newborns with diagnosis of congenital diaphragmatic hernia were included. They presented birth weight from 2.38 to $3.45 \mathrm{~kg}$, gestational age between 36 and 39 weeks; five of them were males. All infants were intubated at delivery within the first minute of life. The surgery was performed between the $2^{\text {nd }}$ and the $6^{\text {th }}$ days of life, and a patch
Instituição: Hospital Israelita Albert Einstein (HIAE), São Paulo, SP, Brasil ${ }^{1}$ Mestre em Pediatria pela Faculdade de Medicina da USP (FMUSP), médico Neonatologista da Unidade de Terapia Intensiva Neonatal (Utin) do HIAE, São Paulo, SP, Brasil

${ }^{2}$ Médico Neonatologista da Utin do HIAE, São Paulo, SP, Brasil ${ }^{3}$ Coordenadora Médica da Unidade Neonatal do HIAE, encarregada do Berçário Anexo à Maternidade do Instituto da Criança do Hospital das Clínicas da FMUSP, doutora em Medicina pela FMUSP, professora colaboradora do Departamento de Pediatria da FMUSP, São Paulo, SP, Brasil

${ }^{4}$ Professor livre-docente em Pediatria pela FMUSP, coordenador médico do Centro de Terapia Intensiva Pediátrica do HIAE, São Paulo, SP, Brasil
${ }^{5}$ Doutor em Pediatria pela FMUSP, médico Neonatologista da Utin do HIAE, professor colaborador do Departamento de Pediatria da Faculdade de Medicina da Universidade de São Paulo (FMUSP), coordenador da Unidade de Pesquisa Experimental do ICr/HC-FMUSP, São Paulo, SP, Brasil

Endereço para correspondência:

Celso Moura Rebello

Rua Engenheiro Teixeira Soares, 165

CEP 05505-030 - São Paulo/SP

E-mail: celso.rebello@uol.com.br

Recebido em: 21/4/2008

Aprovado em: 26/7/2008 
was needed in four patients. A "gentle" ventilation strategy was used in six infants, targeting normal pre-ductal $\mathrm{PaO}_{2}$ and allowing hypercapnia $\left(\mathrm{PaCO}_{2}\right.$ between 50 and $\left.60 \mathrm{mmHg}\right)$. The extubation occurred between the $1^{\text {st }}$ and $12^{\text {th }}$ day after surgery, except for one infant who died. Six newborns were discharged with an average post-natal age of 30 days (19 to 55 days). The survival rate was $75 \%$.

Conclusions: The systematic care of infants with diagnosis of congenital diaphragmatic hernia can assure a survival rate comparable to reference centers.

Key-words: hernia, diaphragmatic; respiration, artificial; infant, newborn.

\section{Introdução}

Apesar da disponibilidade de recursos como ventilação de alta freqüência, óxido nítrico e uso de membrana de oxigenação extracorpórea (ECMO), a mortalidade associada à hérnia diafragmática congênita é elevada, ocorrendo em 37 a 47\% dos casos, mesmo em centros de alta complexidade ${ }^{(1,2)}$. A hipoplasia pulmonar, a lesão pulmonar associada à ventilação mecânica e à expansão pulmonar ipsilateral muito rápidas contribuem para a mortalidade ${ }^{(3)}$.

Em nosso meio há relatos de séries de casos com morbimortalidade ainda extremamente elevada ${ }^{(4)}$. O presente estudo visa avaliar o emprego de uma abordagem ventilatória conservadora e descrever a evolução de oito pacientes com diagnóstico de hérnia diafragmática congênita admitidos na Unidade de Tratamento Intensivo Neonatal (Utin) do Hospital Israelita Albert Einstein (HIAE) nos períodos pré e pós-operatório.

\section{Método}

Estudou-se a coorte histórica dos pacientes com diagnóstico de hérnia diafragmática congênita, nascidos no período de junho de 2001 a novembro de 2006, sendo que o acesso aos prontuários contou com a anuência do Comitê de Ética em Pesquisa do HIAE.

Avaliaram-se os dados referentes ao recém-nascido (idade gestacional, peso ao nascer, via de parto, sexo, escore de Apgar e presença de malformações associadas), à reanimação, à estabilização pré-operatória (via de intubação, dados de oxigenação das primeiras 24 horas de vida, modo de ventilação, uso de drogas e presença de hipertensão pulmonar avaliada em todos os pacientes por ecocardiografia), aos cuidados no intra e pós-operatório (hemorragia com necessidade de transfusão, drenagem pleural, necessidade de tela (patch), uso de óxido nítrico, administrado de acordo com a repercussão clínica da hipertensão pulmonar, com necessidade de $\mathrm{FiO}_{2} \geq 0,4$, tempo de ventilação mecânica e de internação, sobrevida e morbidade associada). Também foram obtidos dados referentes aos fatores prognósticos na ultra-sonografia pré-natal (relação pulmão fetal/circunferência cefálica e presença de fígado intratorácico). Os dados foram coletados para análise descritiva, não sendo constituído um grupo de comparação com estratégia ventilatória agressiva. Foram incluídos todos os recém-nascidos com diagnóstico de hérnia diafragmática congênita nascidos a partir de junho de 2001, época em que foi adotada a estratégia ventilatória conservadora em pacientes com hérnia diafragmática congênita.

No período analisado foi adotada uma estratégia ventilatória que visa a proteger os pulmões. Esta estratégia inclui intubação imediata após o nascimento, início da descompressão gástrica (aspiração contínua) desde a sala de parto, cateterização dos vasos umbilicais logo após a admissão na Utin, sedação leve sem curarização, uso de ventilação convencional inicialmente no modo assistido/controlado, seguido de ventilação mandatória intermitente sincronizada associada à pressão de suporte. Em todos os pacientes procurou-se adotar uma estratégia ventilatória com hipercapnia permissiva $\left(\mathrm{PaCO}_{2}\right.$ entre 50 e $60 \mathrm{mmHg}$, mantendo-se $\left.\mathrm{pH}>7,24\right)$ com valores-alvo para a saturação de oxigênio pré-ductal entre 89 e $94 \%$.

Foi realizado o ecocardiog rama nos oito pacientes para o diagnóstico de hipertensão pulmonar, sendo indicado óxido nítrico diante da necessidade de $\mathrm{FiO}_{2}$ acima de $40 \%$ associado à hipertensão pulmonar. A dose inicial de óxido nítrico foi de $20 \mathrm{ppm}$, com manutenção desta até a redução da $\mathrm{FiO}_{2}$ abaixo do valor de introdução e estabilidade clínica. O desmame do gás foi feito em intervalos de 5 ppm a cada 12 horas, até 5 ppm; em seguida, tentou-se uma redução de 1 a 2 ppm a cada 12 horas até a suspensão completa. Foi associado sildenafil $(0,4 \mathrm{mg} / \mathrm{kg} / \mathrm{dose}$, a cada seis horas) nos neonatos que estavam recebendo óxido nítrico, mas não mostravam estabilização clínica (quedas freqüentes de saturação) e/ou com impossibilidade de se reduzir a $\mathrm{FiO}_{2}$. A correção cirúrgica foi realizada após o controle da hipertensão pulmonar, evitando, dessa forma, a drenagem pleural profilática no intra-operatório e permitindo a expansão lenta do pulmão ipsilateral à hérnia diafragmática congênita. 


\section{Resultados}

Foram identificados oito pacientes com diagnóstico antenatal da hérnia. Os dados de ultra-sonografia pré-natal foram obtidos em sete pacientes, sendo que em quatro deles houve descrição dos fatores prognósticos pesquisados (Tabela 1). Os dados referentes à idade gestacional, à via de parto, ao peso ao nascimento, ao sexo, ao boletim de Apgar no primeiro e quinto minutos e às malformações associadas estão descritos na Tabela 2.

Todos os pacientes receberam descompressão gástrica desde a sala de parto. Com exceção do paciente número 2 (portador de fenda palatina, com necessidade de mais de uma tentativa), todos foram intubados logo após o nascimento (os pacientes 1 e 3 foram intubados por via nasotraqueal e os demais por via orotraqueal).

Para a ventilação, foi empregado o modo assistido/controlado ou ventilação mandatória intermitente sincronizada (SIMV) associada à pressão de suporte. Em nenhum neonato foi aplicada a ventilação de alta freqüência. O surfactante exógeno foi administrado apenas no paciente 5 (Survanta ${ }^{\circledR}$, $100 \mathrm{mg} / \mathrm{kg}$ na terceira hora de vida). Os valores da relação $\mathrm{PaO}_{2} / \mathrm{FiO}_{2}$, os valores máximos e mínimos da $\mathrm{PaCO}_{2}$ e do índice de oxigenação ( $\mathrm{IO}=$ pressão média de vias aéreas versus $\mathrm{FiO}_{2} / \mathrm{PaO}_{2}$ ) e a relação do maior índice de oxigenação e a $\mathrm{PaCO}_{2}$ nas primeiras 24 horas de vida são descritos na Tabela 3. O óxido nítrico foi utilizado no período pré-operatório apenas nos pacientes 4 e 8 , sendo que o último faleceu antes da correção cirúrgica, enquanto o primeiro recebeu sildenafil associado à partir do terceiro dia de vida.

Os recém-nascidos foram operados, em média, com $3,4 \pm 1,5$ dias de vida. Todas as hérnias estavam localizadas à esquerda do diafragma sendo que, durante a correção cirúrgica, apenas os pacientes 2 e 5 necessitaram de transfusão. $\mathrm{O}$ paciente 2 foi o único a receber drenagem profilática intraoperatória do hemitórax ipsilateral à hérnia. Foi necessário o uso de tela para a correção da hérnia em quatro pacientes, o que equivale a $57 \%$ dos operados.

No pós-operatório, os pacientes 2 e 4 fizeram uso de óxido nítrico, o primeiro por mais de um mês e o segundo tanto na estabilização inicial como após a correção cirúrgica. O paciente 4 recebeu novamente sildenafil, mantendo seu uso por via oral até a alta hospitalar devido à dificuldade de suspensão do óxido nítrico. Os recém-nascidos foram extubados, em média, no dia 4,8 4 ,1 do pós-operatório e receberam alta hospitalar com 30,3 $\pm 15,6$ dias de vida. Nessa casuística alcançou-se sobrevida de $75 \%$ em todos os recém-nascidos e de $85,7 \%$ nos que receberam estabilização inicial e foram submetidos à correção cirúrgica (Tabela 3).

Houve necessidade de novas internações para intervenção cirúrgica no local da correção da hérnia diafragmática congênita com tela no paciente 4 . Não houve descrição de doença pulmonar crônica entre os estudados.

Tabela 1 - Dados prognósticos presentes na ultra-sonografia pré-natal

\begin{tabular}{lcccc}
\hline Paciente & $\begin{array}{c}\text { Idade gestacional } \\
\text { (semanas) }\end{array}$ & Polidrâmnio & LHR & Fígado intratóracico \\
\hline 1 & 27 & Não & Não referido & Não \\
3 & 26 & Sim (ILA=26) & 1,03 & Não \\
4 & 20 & Não & 1,37 & Não \\
5 & 26 & Não & Não referido & Não \\
6 & 26 & Sim (ILA=21) & Não referido & Não \\
7 & 26 & Não & 2,27 & Não \\
8 & 26 & Não referido & 0,7 & Sim \\
\hline
\end{tabular}

LHR: relação pulmão/circunferência cefálica; ILA: índice de líquido amniótico.

Tabela 2 - Características de nascimento dos pacientes com Hérnia Diafragmática Congênita

\begin{tabular}{lcccccc}
\hline Paciente & $\begin{array}{c}\text { I.G. } \\
\text { (semanas) }\end{array}$ & Via parto & Peso $(\mathbf{g})$ & Sexo & $\begin{array}{c}\text { Apgar 1\% } \\
\text { Apgar 5 } \text { \ }^{\text {minuto }}\end{array}$ & $\begin{array}{c}\text { Malformações } \\
\text { associadas }\end{array}$ \\
\hline 1 & 38 & Cesárea & 3430 & $\mathrm{M}$ & $7 / 9$ & Não \\
2 & 38 & Cesárea & 2490 & $\mathrm{M}$ & $8 / 8$ & Sim* \\
3 & 38 & Cesárea & 3050 & $\mathrm{M}$ & $3 / 6$ & Não \\
4 & 37 & Cesárea & 2890 & $\mathrm{~F}$ & $8 / 9$ & Não \\
5 & 38 & Cesárea & 2785 & $\mathrm{~F}$ & $3 / 9$ & Não \\
6 & 36 & Normal & 2890 & $\mathrm{~F}$ & $5 / 9$ & Não \\
7 & 39 & Normal & 3450 & $\mathrm{M}$ & $8 / 9$ & Não \\
8 & 39 & Cesárea & 2380 & $\mathrm{M}$ & $4 / 6$ & Sim \\
\hline
\end{tabular}

*Fenda palatina e hipospádia; §múltiplas comunicações inter-ventriculares. 
Tabela 3 - Características pré, intra e pós-operatórias dos pacientes com hérnia diafragmática congênita

\begin{tabular}{lccccccc}
\hline Paciente & $\mathrm{PaO}_{2} / \mathrm{FiO}_{2}{ }^{*}$ & $\begin{array}{c}\text { Dia } \\
\text { cirúrgico }\end{array}$ & $\begin{array}{c}\text { Óxido } \\
\text { nítrico }\end{array}$ & $\begin{array}{c}\text { Uso de } \\
\text { tela }\end{array}$ & $\begin{array}{c}\text { Dreno } \\
\text { torácico }\end{array}$ & $\begin{array}{c}\text { Extubação } \\
\text { (pós-operório) }\end{array}$ & $\begin{array}{c}\text { Dia de } \\
\text { alta }\end{array}$ \\
\hline 1 & 153,8 & 2 & $\mathrm{Não}$ & $\mathrm{Não}$ & Não & 1 & 19 \\
2 & 187,8 & 6 & $\mathrm{Sim}{ }^{* *}$ & $\mathrm{Sim}$ & $\mathrm{Sim}$ & $76^{* *}$ & 82 (óbito) \\
3 & 91,0 & 3 & $\mathrm{Não}$ & $\mathrm{Sim}$ & Não & 2 & 45 \\
4 & 82,6 & 5 & $\mathrm{Sim}{ }^{\S}$ & $\mathrm{Sim}$ & Não & 12 & 55 \\
5 & 185,7 & 3 & $\mathrm{Não}$ & Não & Não & 7 & 20 \\
6 & 374,3 & 3 & Não & Sim & Não & 3 & 22 \\
7 & 212,0 & 2 & Não & Não & Não & 4 & 21 \\
8 & 59,0 & - & Sim & - & - & - & 2 (óbito) \\
\hline
\end{tabular}

*melhor relação $\mathrm{PaO}_{2} / \mathrm{FiO}_{2}$ nas primeiras 24 horas de vida; ** paciente permaneceu com uso de óxido nítrico até o óbito, após a correção cirúrgica; spaciente fez uso também de sildenafil, tanto no pré como no pós-operatório.

\section{Discussão}

O avanço mais importante nos cuidados ao paciente com hérnia diafragmática congênita nos últimos 20 anos foi a propagação da idéia da ventilação protetora (gentle-ventilation) $)^{(5)}$, em contraste com a hiperventilação, modo bastante popular até a década de $1980^{(6)}$. A lesão secundária ao estresse pulmonar gerado pela hiperventilação, que buscava reverter a hipertensão pulmonar à custa de alcalose respiratória e hiperóxia, causa alterações no pulmão remanescente com formação de membranas hialinas e áreas hemorrágicas, possibilitando o surgimento de barotrauma, iatrogenia responsável por até $25 \%$ das mortes nesses $\operatorname{casos}^{(1,2)}$.

A ventilação protetora limita os picos de pressão empregados, permitindo hipercapnia e uma relativa hipoxemia, com redução da agressão pulmonar, além de manter a respiração espontânea do paciente ${ }^{(3,5,7)}$. Seu uso permitiu reduzir a freqüência de pneumotórax nos pacientes ventilados de $83 \%$ (com a hiperventilação) para $1,9 \%$, além de diminuir a mortalidade intra-hospitalar ${ }^{(8)}$. A ventilação oscilatória de alta freqüência, não utilizada nos pacientes aqui relatados, tem sido defendida por alguns autores, principalmente diante de hipoxemia refratária, mas não há estudos que comprovem sua superioridade em relação à ventilação convencional protetora $^{(1,9)}$. Na verdade, há grande semelhança entre a ventilação convencional, que emprega volumes correntes reduzidos e mantém a estabilidade alveolar, quando comparada à ventilação de alta freqüência oscilatória.

Embora empregadas nesta série de casos, outras estratégias utilizadas para o tratamento da insuficiência respiratória associada à hérnia diafragmática congênita, como o óxido nítrico inalatório e o sildenafil, não mostram a mesma consistência dos resultados obtidos com a ventilação protetora. O uso do óxido nítrico pode ocasionar uma breve e transitória estabilização do recém-nascido, mas não traz maior sobrevida aos pacientes. Seu uso é defendido como estratégia de transição para estabilizar o paciente que será encaminhado à $\mathrm{ECMO}^{(8,10)}$. Agindo também na promoção da vasodilatação pulmonar, o sildenafil (inibidor da fosfodiesterase) foi utilizado em um dos pacientes desta série devido à dificuldade de redução da concentração de óxido nítrico; a administração de sildenafil na hérnia diafragmática congênita conta apenas com relatos esporádicos em literatura, embora seu custo seja inferior ao do óxido nítrico inalatório ${ }^{(11,12)}$. O surfactante exógeno, utilizado nas primeiras horas de vida em um dos recém-nascidos estudados, também não conta com evidências científicas para seu uso regular em portadores de hérnia diafragmática congênita ${ }^{(8,13)}$.

Todos foram operados após a estabilização da hipertensão pulmonar, de acordo com a tendência observada na literatura internacional que tem orientado a adoção de correção cirúrgica eletiva para os pacientes com a hérnia ${ }^{(6,9,14)}$. A necessidade de uso de tela para a correção diafragmática não levou a maiores complicações na casuística apresentada, embora seja um dado ainda conflitante na literatura ${ }^{(1,2)}$. Apenas um paciente recebeu drenagem pulmonar durante o intra-operatório, sendo que também apresentava fígado intratorácico. $\mathrm{O}$ mesmo neonato mostrou-se instável no pré-operatório, após um período de "lua-de-mel", sendo o único que evoluiu a óbito. A drenagem pulmonar profilática é também descrita como fator de maior mortalidade em pacientes operados por hérnia diafragmática congênita ${ }^{(3,7)}$.

A sobrevida encontrada nos pacientes estudados foi de $75 \%$, incluindo-se o caso de óbito precoce cujos dados antenatais estimavam risco de morte de $100 \%$. Considerando-se os pacientes operados, a sobrevida foi de $85,7 \%$, comparável a centros especializados no cuidado a neonatos com hérnia 
diafragmática congênita do Japão ${ }^{(9)}$, Europa ${ }^{(15)}$ e América do Norte (1-3,7). $^{(1)}$.

É importante salientar que este estudo apresenta uma série de limitações, incluindo o pequeno número de casos e o fato de se tratar de um estudo retrospectivo. Embora no período analisado a abordagem ventilatória tenha sido feita de modo padronizado, não foi constituído um grupo controle com ventilação convencional mais agressiva, o que tornaria possível verificar com maior clareza o papel da estratégia protetora nos resultados encontrados. Outra limitação se refere à dificuldade de definição da gravidade dos casos relatados nesta série. Apesar de a relação pulmão/circunferência cefálica, o índice de líquido amniótico e a presença de fígado intratorácico indicarem que, em pelo menos um terço dos casos os recém-nascidos apresentavam um prognóstico mais reservado, o grau de hipoplasia pulmonar não pôde

\section{Referências bibliográficas}

1. Azarow K, Messineo A, Pearl R, Filler R, Barker G, Bohn D. Congenital diaphragmatic hernia: a tale of two cities: the Toronto experience. J Ped Surg 1997;32:395-400.

2. Wilson JM, Lund DP, Lillehei CW, Vacanti JP. Congenital diaphragmatic hernia: a tale of two cities: the Boston experience. J Ped Surg 1997;32:401-5.

3. Boloker J, Bateman DA, Wung JT, Stolar CJ. Congenital diaphragmatic hernia in 120 infants treated consecutively with permissive hypercapnea/spontaneous respiration/elective repair. J Ped Surg 2002;37:357-66.

4. Ruano R, Bunduki V, Silva MM, Yoshizaki CT, Tanuri U, Macksoud JG et al. Prenatal diagnosis and perinatal outcome of 38 cases with congenital diaphragmatic hernia: 8-year experience of a tertiary Brazilian center. Clinics 2006;61:197-202.

5. Wung JT, James LS, Kilchevsky E, James E. Management of infants with severe respiratory failure and persistance of the fetal circulation, without hyperventilation. Pediatrics 1985;76:488-94.

6. Fox WW, Duara S. Persistent pulmonary hypertension in the neonate: diagnosis and management. J Pediatr 1983;103:505-14.

7. Wung JT, Sahni R, Moffitt ST, Lipsitz E, Stolar CJ. Congenital diaphragmatic hernia: survival treated with very delayed surgery, spontaneous respiration, and no chest tube. J Ped Surg 1995;30:406-9.

8. Kays DW, Langham MR Jr, Ledbetter DJ, Talbert JL. Detrimental effects of standard medical therapy in congenital diaphragmatic hernia. Ann Surg 1999;230:340-51. ser estimado com precisão. Tais limitações não permitem que, isoladamente, os resultados encontrados possam ser generalizados, mas eles somam mais evidências à literatura quanto aos benefícios de uma estratégia ventilatória protetora na abordagem da hérnia diafragmática congênita. Os autores concluem, diante da série de casos apresentada, que a adoção de estratégia ventilatória protetora para o manuseio de recém-nascidos com hérnia diafragmática congênita provavelmente contribui para uma sobrevida comparável à descrita nos principais centros mundiais.

\section{Agradecimentos}

Agradecemos às doutoras Denise Lapa Pedreira e Sineida Aparecida Rodrigues Girão pelo auxílio na obtenção de dados pré-natais dos pacientes estudados.

9. Okuyama H, Kubota A, Oue T, Kuroda S, Ikegami R, Kamiyama M et al Inhaled nitric oxide with early surgery improves the outcome of antenatally diagnosed congenital diaphragmatic hernia. J Pediatr Surg 2002;37: 1188-90.

10. No authors listed.Inhaled nitric oxide and hypoxic respiratory failure in infants with congenital diaphragmatic hernia. The Neonatal Inhaled Nitric Oxide Study Group (NINOS). Pediatrics 1997;99:838-45.

11. Sydorak RM, Harrison MR. Congenital diaphragmatic hernia: advances in prenatal therapy. Clin Perinatol 2003;30:465-79.

12. Oliveira EC, Oliveira AM, Amaral CF, Oliveira JS, Miranda ME, Pontes AK. Uso de Sildenafil para facilitar a retirada de óxido nítrico inalado em pós-operatório de hérnia diafragmática: Relato de caso. RBTI 2005;17:60-2.

13. Van Meurs K; Congenital Diaphragmatic Hernia Study Group. Is surfactant therapy beneficial in the treatment of the term newborn infant with congenital diaphragmatic hernia? J Pediatr 2004;145:312-6.

14. dos Santos LR, Maksoud-Filho JG, Tannuri U, Andrade WC, Maksoud JG. Prognostic factors and survival in neonates with congenital diaphragmatic hernia. J Pediatr (Rio J) 2003;79:81-6.

15. Bétrémieux $P$, Lionnais $S$, Beuchée A, Pladys $P$, LeBouar $G$, Pasquier L et al. Perinatal management and outcome of prenatally diagnosed congenital diaphragmatic hernia: a 1995-2000 series in Rennes University Hospital. Prenat Diagn 2002;22:988-94. 